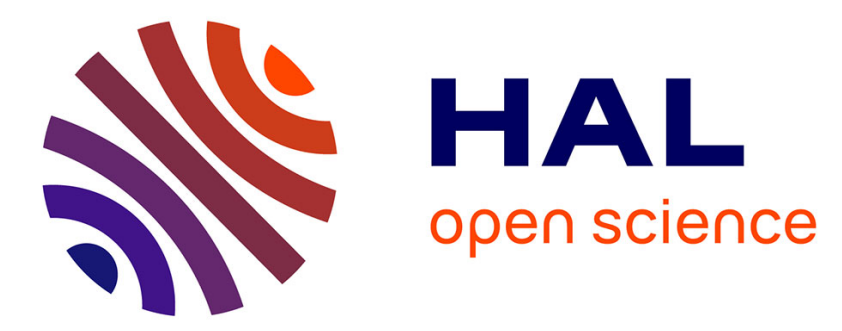

\title{
Sensitivity analysis of complex models: coping with dynamic and static inputs
}

Floriane Anstett-Collin, Jeanne Goffart, Thierry A. Mara, Lilianne

Denis-Vidal

\section{- To cite this version:}

Floriane Anstett-Collin, Jeanne Goffart, Thierry A. Mara, Lilianne Denis-Vidal. Sensitivity analysis of complex models: coping with dynamic and static inputs. Reliability Engineering and System Safety, 2015, 134, pp.268-275. 10.1016/j.ress.2014.08.010 . hal-01063795

\section{HAL Id: hal-01063795 https://hal.science/hal-01063795}

Submitted on 13 Sep 2014

HAL is a multi-disciplinary open access archive for the deposit and dissemination of scientific research documents, whether they are published or not. The documents may come from teaching and research institutions in France or abroad, or from public or private research centers.
L'archive ouverte pluridisciplinaire HAL, est destinée au dépôt et à la diffusion de documents scientifiques de niveau recherche, publiés ou non, émanant des établissements d'enseignement et de recherche français ou étrangers, des laboratoires publics ou privés. 


\title{
Sensitivity analysis of complex models: coping with dynamic and static inputs
}

\author{
F. Anstett-Collin ${ }^{\mathrm{a}}$, J. Goffart $^{\mathrm{b}}$, T. Mara ${ }^{\mathrm{c}}$, L. Denis-Vidal $^{\mathrm{d}}$ \\ ${ }^{a}$ University of Lorraine, CRAN UMR 7039, 2 rue Jean Lamour, 54519 Vandoeuvre Les \\ Nancy, France. Email: floriane.collin@univ-lorraine.fr \\ ${ }^{b}$ University of Savoy, LOCIE, Le Bourget du Lac, France. Email: \\ jeanne.goffart@univ-savoie.fr \\ ${ }^{c}$ University of Reunion Island, PIMENT/MASC, Department of Physics, UFR ST, 15 \\ Avenue Rene Cassin, 97715 Saint-Denis La Reunion, France. Email: \\ thierry.mara@univ-reunion.fr \\ ${ }^{d}$ University of Compiègne, LMAC, Centre de Recherches de Royallieu BP 20 529, 60205 \\ Compiègne Cedex, France. Email: lilianne.denis-vidal@math.univ-lille1.fr
}

\begin{abstract}
In this article, we address the issue of conducting a sensitivity analysis of complex models with both static and dynamic uncertain inputs. While several approaches have been proposed to compute the sensitivity indices of the static inputs (i.e. parameters), the ones of the dynamic inputs (i.e. stochastic fields) have been rarely addressed. For this purpose, we first treat each dynamic as a Gaussian process. Then, the truncated Karhunen-Loève expansion of each dynamic input is performed. Such an expansion allows to generate independent Gaussian processes from a finite number of independent random variables. Given that a dynamic input is represented by a finite number of random variables, its variance-based sensitivity index is defined by the sensitivity index of this group of variables. Besides, an efficient samplingbased strategy is described to estimate the first-order indices of all the input factors by only using two input samples. The approach is applied to a build-
\end{abstract}


ing energy model, in order to assess the impact of the uncertainties of the material properties (static inputs) and the weather data (dynamic inputs) on the energy performance of a real low energy consumption house.

Keywords: Global sensitivity analysis, dynamic and static inputs, Karhunen-Loève expansion, building energy model

\section{Introduction}

Nowadays, one important feature when designing high-performance buildings is to reduce the energy consumption, for both economical and environmental purposes. Thus, the need for a better control and optimization of the energy consumption of buildings and houses arises since last three decades. In this framework, softwares for thermal building performance simulation have been developed and are constantly updating due to the evolution of construction techniques and materials. This leads to complex, high-dimensional and multi-physics models, presenting uncertain inputs due to measurements, expert judgements or simply ignorance. Thus, the problem of the reliability of these models arises. Sensitivity Analysis (SA) can help evaluating the impact of this lack of knowledge onto the model responses. SA aims at determining the most influential input factors onto the model responses. Numerous studies, in many different application fields (see for example $[1,2,3,4,5,6,7]$ ), have focused on the sensitivity analysis of models with static inputs (i.e. parameters). Global SA is generally preferred because the input factors are varied simultaneously in a large uncertainty range and can reveal possible interactions between the inputs. Global SA are often based on the analysis of the model response variance and are known as ANOVA (ANalysis Of 
VAriance) methods $[8,9]$.

Regarding building energy modeling, existent surveys have only analysed the influence of static inputs onto building models, such as the effects of the materials thermophysical properties [10]. However, some phenomena previously neglected for energy-consuming building may become preponderant in the consumption of low energy buildings ${ }^{1}$, as the consideration of weather data variability, not only the temperature but also the solar radiation, the relative humidity, the wind speed, etc. Indeed, it is expected that meteorological inputs play a crucial role in designing high-performance buildings. There are very few studies that try to assess the influence of the weather inputs because they are time-dependent (dynamic inputs). This can be explained by the fact that considering coherent long-term "typical" weather data is not obvious. As stated in [11], if the weather data, usually on an hourly basis are available, there is no need to simulate them. However, when the hourly data are lacking, there is a real need to simulate weather sequences. Even if they are available, sometimes it might only represent a few years of record which is anecdotal and cannot represent long-term typical weather. The only

\footnotetext{
${ }^{1}$ The term "low energy consumption building" refers to standard for energy efficiency in buildings. This eco-label is assigned to buildings satisfying some standards, mainly related to the annual total primary energy consumption of the building. In France, the average annual requirement for heating, cooling, ventilation, hot water and lighting must be lower than $50 \mathrm{kWh} / \mathrm{m}^{2}$ (in primary energy) for new buildings and lower than $80 \mathrm{KWh} / \mathrm{m}^{2}$ for renovations. These limits are set by the French Ministry for Ecology, Energy, Sustainable Development and Territorial Development, according to the European Commission policy (see http://www.europarl.europa.eu/committees/en/home.html). The values can differ from one European country to the other, depending on their climate.
} 
way to represent the full spectrum of weather conditions is to collect data from many years of hourly weather data. Very few weather sites have reliable contiguous weather data available for extremely long periods of time. Thus, an alternative is to simulate hourly weather data using a model. But, it is not straightforward to generate time series that satisfy the desired random field distribution.

The aim of this study is to assess the impact of uncertain inputs, such as the weather data and the thermophysical properties of materials, onto the heat consumption of an actual high-energy performance house. This problem is challenging because it implies to perform the sensitivity analysis of a time consuming computer model with uncertain static and spatio/temporal inputs. Such an issue is rarely addressed in the literature (for instance, in [12]). The uncertain static inputs are treated as independent random variables defined by their marginal distribution while the dynamic inputs are treated as Gaussian processes (GP) defined by a temporal mean and a covariance function. In a Monte Carlo approach, sampling the independent static inputs can be achieved with a space filling sampler such as the Latin Hypercube sampling [13] or a $\operatorname{LP} \tau$ sequence [14]. It is more challenging to generate Gaussian processes and especially to assess their influence.

A GP can be represented as a series expansion involving a complete set of deterministic functions with corresponding random coefficients [15, 16], as the truncated Karhunen-Loève (KL) expansion [17, 18]. KL series expansion is based on the eigen-decomposition of the covariance function, involving orthogonal deterministic basis functions and the orthogonal uncorrelated random coefficients. This allows the optimal encapsulation of the informa- 
tion contained in the GP into a set of discrete independent random variables. The GP can then be represented by a linear combination of the deterministic functions depending on time whose coefficients are independent random variables. For sensitivity analysis purposes, the influence of the dynamic inputs onto the model response is then given by the one of these random coefficients of the Karhunen-Loève decomposition, which is the originality of the present paper.

The paper is organized as follows. In Section 2, SA for static inputs is briefly recalled and then extended to inputs depending on time. The procedure to carry out a SA of model with uncertain static and dynamic inputs is provided. In section 3 , the proposed approach is applied to a building energy model to quantify the impact of each weather data input and each thermophysical material properties on the energy performance of a real low-energy consumption house.

\section{Sensitivity analysis}

\subsection{Model with static inputs}

First, let us recall that variance-based sensitivity index is computed when the model is only function of static inputs. Consider the following model:

$$
\boldsymbol{y}(\theta)=g\left(\boldsymbol{\omega}^{s}(\theta)\right)
$$

where $\boldsymbol{y}=\left\{y_{1}, \ldots, y_{m}\right\}$ is the set of $m$ model responses of interest, $g$ the model function which is known, $\boldsymbol{\omega}^{s}=\left\{\omega_{1}^{s}, \ldots, \omega_{N_{s}}^{s}\right\}$ the set of $N_{s}$ uncertain static inputs, $\theta \in \Omega$ where $\Omega$ is the space of random events. The stochastic variable $\theta$ represents the elementary event of a probability space. It is used 
to indicate the randomness of the input and the output. In the following, we assume that random variables $\omega_{i}^{s}$ are independent and defined by their marginal distribution.

The effect of the uncertain inputs $\omega_{i}^{s}$ onto $y_{j}$ can be assessed by its first-order sensitivity index given by:

$$
S_{i}^{j}=\frac{V\left(E\left(y_{j} \mid \omega_{i}^{s}\right)\right)}{V\left(y_{j}\right)}
$$

where $V\left(E\left(y_{j} \mid \omega_{i}^{s}\right)\right)$ is the variance of the conditional expectation of $y_{j}$ when $\omega_{i}^{s}$ is set and $V\left(y_{j}\right)$ is the total variance of the output $y_{j}$. The first order sensitivity index $S_{i}$ represents the amount of variance of $y_{j}$ due to $\omega_{i}^{s}$ alone. The value of $S_{i}^{j}$ lies between 0 and 1 . The closer to 1 its value is, the more input $\omega_{i}^{s}$ contributes to the total variance of the output. In particular, when $\sum_{1=1}^{N_{s}} S_{i}^{j} \sim 1$ the relationship between $y_{j}$ and the inputs $\boldsymbol{\omega}^{s}$ is additive. Then, $S_{i}^{j}$ can be used to rank the inputs by order of importance. Sensitivity indices of higher order can also be computed to assess the influence of the input interactions but it is not presented here, see [1] for more details.

The definition of the variance-based sensitivity index of one single input variable Eq.(2) is easily extended to the sensitivity index of the group of input variables $\boldsymbol{\omega}_{\boldsymbol{u}}^{\boldsymbol{s}} \subset\left\{\omega_{1}^{s}, \ldots, \omega_{N_{s}}^{s}\right\}$ (also called the closed-order effect),

$$
S_{\mathbf{u}}^{j}=\frac{V\left(E\left(y_{j} \mid \boldsymbol{\omega}_{u}^{s}\right)\right)}{V\left(y_{j}\right)}
$$

\subsection{Estimating the sensitivity indices with two LHS samples}

Some models may be complex with a high number of inputs so that analytical calculations of the previous sensitivity index become time consuming or even impossible. It is therefore necessary to estimate them. For 
this purpose, several approaches have been proposed in the literature (e.g. $[4,19,20,5,21]$, among others). In the present work, the sampling-based method proposed in [22] is employed for two reasons. Firstly, it only requires two Latin Hypercube (LH) samples [13] to estimate all the first-order sensitivity indices. Secondly, it can cope with group of random inputs which is the key of our methodology to estimate the influence of dynamic inputs. Note that, although Quasi Monte Carlo sampling technique is known to have a better coverage of the input space $[14,23]$, LHS is preferred here because we have to deal with a very high number of inputs (thousands) and the sampling design proposed in [22] (similar to rLHS [24]) is suited to LHS.

Let us consider an LH sample $\boldsymbol{\Omega}^{\mathbf{1}}$ of the static inputs of size $N_{s} \times d$ and denote by $\mathbf{Y}^{\mathbf{1}}=g\left(\boldsymbol{\Omega}^{\mathbf{1}}\right)$ the associated model response vector (a vector of size $\left.N_{s} \times m\right)$. We denote by $\mathbf{R}^{1}$ the rank matrix associated with $\boldsymbol{\Omega}^{\mathbf{1}}$. We recall that $\mathbf{R}^{\mathbf{1}}=\left[\mathbf{r}_{1}, \ldots, \mathbf{r}_{d}\right]$ is deduced from $\boldsymbol{\Omega}^{\mathbf{1}}$ by column-wisely replacing the smallest value of $\Omega^{\mathbf{1}}$ by 1 and the highest value by $N_{s}$. As a consequence, an element of $\mathbf{r}_{i}(i=1, \ldots, d)$ is an integer within $\llbracket 1, N_{s} \rrbracket$.

Then, a second input LH sample $\Omega^{\mathbf{2}}$ is generated from $\Omega^{\mathbf{1}}$ by arbitrarily defining an independent rank matrix $\mathbf{R}^{2}$ of size $N_{s} \times d$ and setting,

$$
\Omega^{2}=\Omega^{1} \circ \mathbf{R}^{2}
$$

Note that, the new $\Omega^{2}$ is also an LH sample and this column-wise permutation trick is equivalent to the replicated LH sampling [24]. After running the model with this new sample, a second model response vector $\mathbf{Y}^{\mathbf{2}}=g\left(\boldsymbol{\Omega}^{\mathbf{2}}\right)$ is obtained. The model responses $\mathbf{Y}^{\mathbf{1}}=\left[\mathbf{y}_{1}^{1}, \ldots, \mathbf{y}_{d}^{1}\right]$ and $\mathbf{Y}^{\mathbf{2}}=\left[\mathbf{y}_{1}^{2}, \ldots, \mathbf{y}_{d}^{2}\right]$ allow to estimate the first-order sensitivity index of each static input as fol- 
lows,

$$
\hat{S}_{i}^{j}=\frac{(N-1)^{-1} \sum_{k=1}^{N_{s}}\left(y_{k j}^{r_{i}}-\hat{\mu}_{1}^{j}\right)\left(y_{k j}^{2}-\hat{\mu}_{2}^{j}\right)}{\hat{\sigma}_{1}^{j} \hat{\sigma}_{2}^{j}}
$$

where, $y_{k j}^{r_{i}}$ is $k$-th element of the response vector obtained after reordering the $\mathbf{y}_{j}^{1}(j=1, \ldots, m)$ accordingly with $\mathbf{r}_{i}$, that is,

$$
\mathbf{y}^{r_{i}}=\mathbf{y}_{j}^{1}\left(\mathbf{r}_{i}\right)
$$

The empirical mean of the two response vectors $\mathbf{y}_{j}^{1}$ and $\mathbf{y}_{j}^{2}$ are respectively $\hat{\mu}_{1}^{j}$ and $\hat{\mu}_{2}^{j}$ while $\hat{\sigma}_{1}^{j}$ and $\hat{\sigma}_{2}^{j}$ are their respective standard deviation.

We note that (5) is nothing but a reordering of the response vector $\mathbf{y}_{j}^{1}$ such that compared to $\mathbf{y}_{j}^{2}$ the values of each input variable have been varied except the sample of $\omega_{i}^{s}$ that has been fixed. Finally, if the sensitivity indices of groups of input variables are investigated (say $K$ groups), then $\mathbf{R}^{2}$ is a matrix of size $N_{s} \times K$ and the second sample $\Omega^{2}$ is deduced by column-wisely permuting the values of $\Omega^{1}$ such that the values of the variables in the same group are reordering identically. Equation (4) then estimates closed-order effect of the $i$-th group.

\subsection{Model with static and dynamic inputs}

Consider the following model presenting uncertain static and dynamic inputs:

$$
\mathbf{y}(t, \theta)=g\left(\boldsymbol{\omega}^{\boldsymbol{d}}(t, \theta), \boldsymbol{\omega}^{\boldsymbol{s}}(\theta), t\right)
$$

where $\mathbf{y}$ of components $\left\{y_{1}, \ldots, y_{m}\right\}$ is the vector of $m$ model responses, $t \in \mathcal{D} \subset \mathbb{R}$ the time variable, $\boldsymbol{\omega}^{d}$ of components $\left\{\omega_{1}^{d}, \ldots, \omega_{N_{d}}^{d}\right\}$ the set of $N_{d}$ uncertain dynamic inputs (random fields) depending on time $t$. As previously, $\boldsymbol{\omega}^{s}$ of components $\left\{\omega_{1}^{s}, \ldots, \omega_{N_{s}}^{s}\right\}$ is the set of $N_{s}$ uncertain static 
inputs (random variables). It can be worth noting that in another context, $t$ can also represent the space variable instead of the time. In this case, the following still holds.

As previously, we assume that random variables $\omega_{i}^{s}$ are independent and defined by their marginal distribution. We further assume that the random fields, indexed on a bounded domain $\mathcal{D}$, are Gaussian processes, that is, $\omega_{i}^{d} \sim \mathcal{G P}\left(\bar{\omega}_{i}{ }^{d}(t), C_{i}\left(t_{1}, t_{2}\right)\right), i=1, \ldots, N_{d}$, where $\bar{\omega}_{i}{ }^{d}(t)$ and $C_{i}\left(t_{1}, t_{2}\right)$ are respectively the mean and covariance function. The covariance function is symmetric, positive definite and depends on two different time instants, $t_{1}$ and $t_{2}$, taken here in the form, $C_{i}\left(t_{1}, t_{2}\right)=\sigma_{i}^{2} e^{\eta_{i}\left|t_{2}-t_{1}\right|^{n_{i}}}$.

According to [18], the random field $\omega_{i}^{d}$, can be approximated using the truncated KL series:

$$
\omega_{i}^{d}(t, \theta) \simeq \bar{\omega}_{i}^{d}(t)+\sum_{k i=1}^{M_{i}} \sqrt{\lambda}_{k i} \xi_{k i}(\theta) f_{k i}(t)
$$

where $\lambda_{k i}$ and $f_{k i}$ are the deterministic eigenvalues and eigenfunctions of the covariance function $C_{i}\left(t_{1}, t_{2}\right), \boldsymbol{\xi}_{\boldsymbol{i}}$ is a set of independent standard normal variables and $M_{i}$ is the number of KL-terms. In practice, we retain the first $M_{i}$ eigenmodes that contain the $95 \%$ of the variance of the input $\omega_{i}^{d}$. The number of eigenmodes retained depends on the choice of the covariance function and may be very different from one input to another.

The key feature for simulating random fields using KL expansion lies on the ability to determine accurately the eigenvalues and eigenfunctions of the covariance function. They are given from the spectral decomposition of the 
covariance function $C_{i}\left(t_{1}, t_{2}\right)$, requiring to solve the homogeneous Fredholm integral equation of the second kind given by:

$$
\int_{\mathcal{D}} C_{i}\left(t_{1}, t_{2}\right) f_{k i}\left(t_{1}\right) \mathrm{d} t_{1}=\lambda_{k i} f_{k i}\left(t_{2}\right)
$$

For some covariance functions (first-order Markov or Wiener-Levy processes, for example), the equation (8) can be twice differentiable with respect to $t_{1}$. The resulting differential equation can be solved analytically and eigenvalues can be obtained as well. But in most cases, solving equation (8) requires numerical methods, such as the Galerkin one [25]. To avoid tedious quadratures and alleviating computational effort, an alternative is to use a wavelet-Galerkin approach $[18,26]$. In this case, the representation of integral operators is made in wavelet basis and can be performed without numerical integration. The comparison of wavelet-Galerkin method with other available methods in solving the Fredholm integral equation can be found in $[17]$.

Once the eigenmodes are obtained for all the dynamic inputs $\omega_{i}^{d}(t, \theta)$ using KL decomposition (7), the influence of $\omega_{i}^{d}(t, \theta)$ is then given by the one of the random coefficients $\xi_{k i}(\theta)$ propagated to $\boldsymbol{y}(t, \theta)$. In fact, once the KL decomposition of a given dynamic input $\omega_{i}(t, \theta)$ is performed, the randomness of this dynamic input $\omega_{i}(t, \theta)$ lies on the independent standard normal variables $\xi_{k i}(\theta)$. Thus, the randomness of the dynamic input $\omega_{i}(t, \theta)$ is propagated to the output $y$ through the associated modes $\xi_{k i}$. The influence of the given dynamic input $\omega_{i}(t, \theta)$ on the output $y$ is then represented by the one of all the modes $\xi_{i}$ associated to $\omega_{i}(t, \theta)$ in the KL decomposition. The 
sensitivity index of the modes grouped together represent the influence of the dynamic input $\omega_{i}(t, \theta)$.

Consider the $M_{i}$-dimensional random coefficient $\boldsymbol{\xi}_{\boldsymbol{i}}$, grouping the $M_{i}$ modes of the input $\omega_{i}^{d}$ :

$$
\boldsymbol{\xi}_{i}=\left\{\xi_{1 i}, \ldots, \xi_{M_{i} i}\right\}
$$

Thus, SA of the model output $\boldsymbol{y}(t, \theta)$ is performed through the random vectors $\left\{\boldsymbol{\xi}_{1}, \ldots, \boldsymbol{\xi}_{N_{d}}, \boldsymbol{\omega}^{s}\right\}$, with $\boldsymbol{\xi}_{\boldsymbol{i}}$ given by (9). Consequently, the effect of the group of factors $\boldsymbol{\xi}_{\boldsymbol{i}}$ is the one of the dynamic input $\omega_{i}^{d}$ and so on.

The sensitivity indices of the group of factors $\boldsymbol{\xi}_{i}=\left\{\xi_{1 i}, \ldots, \xi_{M_{i} i}\right\}$ can be computed as:

$$
S_{i}^{j}=\frac{V\left(E\left(y_{j} \mid \xi_{1 i}, \ldots, \xi_{M_{i} i}\right)\right)}{V\left(y_{j}\right)}
$$

where $V\left(E\left(y_{j} \mid \xi_{1 i}, \ldots, \xi_{M_{i}}\right)\right)$ is the variance of the conditional expectation of $y_{j}$ when $\xi_{1 i}, \xi_{2 i}, \ldots$ and $\xi_{M_{i} i}$ are set.

The sensitivity of the static input $\boldsymbol{\omega}^{\boldsymbol{s}}$ can then be computed using (2).

The proposed approach is an alternative to the Pick and Freeze method [27, 28, 29]. However, the Pick and Freeze method is computationally demanding because it requires $N \times\left(N_{d}+1\right)$ model runs where $N_{d}$ is the number of input factors and $N$ is the sample size. In our study, we employ the random balance design trick (also used in replicated Latin Hypercube [24]) that allows to estimate the entire set of first-order sensitivity indices with only two samples of size $N$ (see [22]).

The proposed approach is summed up in the next section. 


\subsection{Summary of the proposed approach}

The computation of the sensitivity indices for model (6) presenting uncertain static and spatio/temporal inputs requires the following steps.

1. Generation of the static inputs

Use a random sampling scheme to generate the $N_{s}$ static inputs $\omega_{i}^{s}$ with the prescribed marginal distribution.

2. Generation of the dynamic inputs

(a) Perform a 2D wavelet transform of $C_{i}\left(t_{1}, t_{2}\right)$, using the Fast Haar wavelet transform algorithm to obtain the eigenvalues $\lambda_{k i}$ and the eigenfunctions $f_{k i}$.

(b) Use a random sampling scheme to generate the independent standard normal variables $\boldsymbol{\xi}_{i}$.

(c) Generate the $N_{d}$ dynamic inputs $\omega_{i}^{d}$ using (7).

3. Computation of the sensitivity indices

(a) Simulate the model with the generated dynamic and static inputs, $\boldsymbol{\omega}^{\boldsymbol{d}}$ and $\boldsymbol{\omega}^{\boldsymbol{s}}$, to obtain the model response $\boldsymbol{y}(t, \theta)$ (eq. 6).

(b) Compute the sensitivity indices of $\left\{\boldsymbol{\xi}_{1}, \ldots, \boldsymbol{\xi}_{N_{d}}, \boldsymbol{\omega}^{s}\right\}$, according to (2) and (10).

In the next section, the proposed approach is applied to a building energy model. 


\section{Application to a building energy model}

\subsection{Building energy model}

The building energy model studied in this work represents a real building located on an experimental platform, introduced by the National Institute of Solar Energy and located in Chambéry, in France. This place presents a temperate continental climate with alpine influence. The building is a single-detached house with low energy consumption (figure 1). The house has double-glazed on each facade except at the north one where it is tripleglazed. The thickness of the wall is $50 \mathrm{~cm}$, made of $15 \mathrm{~cm}$ of perpend, 20 $\mathrm{cm}$ of insulating material and again $15 \mathrm{~cm}$ of perpend. The house contains hundreds of sensors to quantify its thermal behavior. Details about the house can be found in [30] and in the table provided in the appendix.

The building is divided into several thermal zones, but only the living rooms at the ground and the first floors are studied. The solar gains are maximized in winter and minimized in summer thanks to the glazed surfaces distribution and solar shading. The goal of the study is to quantify and characterize the capacity of the building to exploit environmental energy gain, taking into account uncertainties of material properties and natural variability of weather data.

The outputs of interest are energy needs at the ground and at the first floors $(m=2)$ in order to maintain thermal comfort in these rooms. These outputs are the most important ones for the designer to assess the building performance. Very often, the technical choices made during the design stage aim at decreasing the energy need. The model involves 57 uncertain input factors, among them, 51 are static inputs and 6 are dynamic inputs. 
The 51 uncertain static inputs $\left(N_{s}=51\right)$ represent the thermophysical properties of the material as its density, the thermal conductivity, the transmissivity at each facade, the albedo, the airtightness at the different floors, ... They are listed in Appendix A. All the static inputs follow a Gaussian distribution with standard deviation equal to $5 \%$ of the mean value, except for the airtightness. In fact, the airtightness is one of the most uncertain static inputs in the building thermal behavior. Anormal distribution with standard deviation equal to $25 \%$ of the mean value is assumed for this parameter. The six uncertain dynamic inputs $\left(N_{d}=6\right)$ represent the weather data as the outdoor air temperature, the direction and speed of wind, the direct and diffuse radiation and the relative humidity.

A previous study [31], not presented here, has focused on the statistical analysis of the weather data. One month of january representative of typical winter from 20 years of observations has been used, since the heat consumption is the highest and the most costly, during this period of time, at this place. Moreover, considering only one season allows to respect stationarity of data. From the study in [31], the statistical characteristics of a given input, the covariance function and the hourly mean, on one day, has been determined.

From the hourly mean $\bar{\omega}_{i}^{d}$ and the covariance function $C_{i}\left(t_{1}, t_{2}\right)$, the sample generation of the dynamic inputs $\boldsymbol{\omega}^{\boldsymbol{d}}$ can be carried out, as explained in Section 2, using the Fast Haar wavelet algorithm to solve the Fredholm integral (8). To do so, the Matlab software has been used. This leads here to 512 modes for each of the six inputs, that is $M_{i}=512, \forall i=\{1, \ldots, 6\}$. The figures 4 to 9 show, for each dynamic input, the cumulative distribution 
function of the 24 time steps (hours) for every day of january. The left figure shows the cumulative distribution function randomly chosen over the 1000 samples. The right figure shows the cumulative distribution function from the source file used to extract the statistical characteristics of the dynamic inputs.

The 51 uncertain static inputs are generated using Latin Hypercube sampling, according to their prescribed distribution (Gaussian distribution with mean value and standard deviation given in Appendix A). Here, 2 sets of 1000 samples are used to estimate the sensitivity indices (2) and (10), according to the estimator provided in [32]. Then, the model is simulated with the generated static and dynamic inputs, using the dedicated software EnergyPlus [33]. Each simulation lasts 1 minute. The computational time for 2000 simulations is approximatively 32 hours, but with a quad core it can be reduced to 8 hours.

\subsection{Results}

Figure 2 shows the dispersion of the ideal heat consumption, at the ground and first floors. The consumption mean value is $9.25 \times 10^{8} \mathrm{~J}$ with a variance of $9.44 \times 10^{15}$ at the ground floor and $9.32 \times 10^{8} \mathrm{~J}$ with a variance of $4.90 \times 10^{15}$ at the first floor. The heat consumption is almost the same at the ground and first floors but with a slight prevalence of the first floor. The two thermal zones under study have the same surface but there is more glass surface at the ground floor, especially at south facade. Thus, it may be assumed that the solar gain is more important at this floor, during winter.

Figure 3 shows the sensitivity indices at each floor. At the ground floor, the direct solar radiation has the highest sensitivity index (0.48), explaining 
almost $50 \%$ of the heat consumption dispersion. Then, the temperature and the airtightness follow ( 0.30 and 0.13 , respectively). However, for the first floor, the airtightness and the outdoor temperature have almost the same sensitivity index (0.42 and 0.38, respectively), followed by the direct solar radiation (0.21). The airtightness is more influent at the first floor since the direct solar radiation is less influent at this floor. On the other hand, the sensitivity indices of the other parameter can be neglected showing that they are not influential. It is worth noting that these inputs are not influential in the studied model. This conclusion can be different for other models, in the way these variables are taken into account for the calculation of the energy balance. For example, humidity is not relevant here because the ideal heating device considered does not account for the impact of the humidity onto the consumption.

To sum up, at each floor, only 3 inputs among the 57 are influent. Among these 3 inputs, only one static input is influent here (airthightness) and $2 \mathrm{dy}$ namic ones (outdoor air temperature and direct solar radiation), in a different proportion according to the given floor. It can be noticed that the total sensitivity indices are 0.92 for the consumption at the ground floor and 1 for the consumption at the first floor, showing insignificant interaction between inputs.

To conclude, the results highlight the capacity of the passive house to exploit the solar gains during winter, especially at the ground floor. In fact, the solar radiation has a greater impact on a high-insulated passive building than on an energy-consuming one. This is relevant to minimize the heat consumption during winter in this kind of low energy house. 


\section{Conclusion}

In this paper, a method to perform sensitivity analysis of complex models presenting uncertain static and spatio/temporal inputs has been provided. While assessing the influence of a static input of computer models is common, it is an issue to estimate the influence of a dynamic input. For this purpose, the latter is treated as a random process and its eigen-decomposition is performed. This is achieved with the Karhunen-Loève transformation. Then, the influence of the random process of interest is given through the one of its eigenmode variables. Finally, the influence of the random process can be quantified using the well-known Sobol' sensitivity indices for group of variables. Subsequently, a sampling-based strategy has been used to compute the closed-order effect of the group of variables with only two samples.

This approach has been applied to a building energy model. The latter is defined by a set of 51 uncertain parameters and 6 stochastic weather inputs. It was found that only four input factors were relevant for predicting the energy needs of the passive house. In this case, sensitivity analysis can be seen as a tool to help designing strategies to reduce the energy consumption of passive houses.

\section{Acknowledgment}

This work has been supported by French National Research Agency (ANR) through HABISOL program (project FIABILITE $n^{\circ}$ ANR-10-HABISOL004-02) and by the French National Research Center through ASenDyn PEPS project. 


\section{References}

[1] A. Saltelli, K. Chan, and E. M. Scott. Sensitivity Analysis. Wiley, 2004.

[2] G. Blatman and B. Sudret. Sparse polynomial chaos expansion and adaptive stochastic finite element using a regresion approach. Comptes rendus de Mécanique, 336(6):518-523, 2008.

[3] E. Borgonovo. A new uncertainty importance measure. Reliability Engineering E3 System Safety, 92:771-784, 2007.

[4] R. I. Cukier, R. I. Levine, and K. E. Shuler. Nonlinear sensitivity analysis of multiparameter model systems. Journal of Computational Physics, 26:1-42, 1978.

[5] H.C. Frey and S. R. Patil. Identification and review of sensitivity analysis methods. Risk Analysis, 22(3):553-578, 2002.

[6] I. M. Sobol. Sensitivity estimates for nonlinear mathematical models. Mathematical Modelling and Computing Experiments, 1:407-414, 1993.

[7] R. Kiebre, F. Collin, and M. Basset. Sensitivity analysis for the study of influential parameters in tyre models. International Journal of Vehicle Systems Modelling and Testing, 6(1):72-87, 2011.

[8] T. Homma and A. Saltelli. Importance measures in global sensitivity analysis of model output. Reliability Engineering 83 System Safety, 52(1):1-17, 1996. 
[9] A. Saltelli, S. Tarantola, and K. Chan. A quantitative model independent method for global sensitivity analysis of model output. Technometrics, 41:39-56, 1999.

[10] W. Tian. A review of sensitivity analysis methods in building energy analysis. Renewable and Sustainable Energy Reviews, 20:411-419, 2013.

[11] L. Degelman. Simulation and uncertainty: weather predictions. In A. M. Malkawi and G. Augenbroe, editors, Advanced Building Simulation. Taylor and Francis, 2004.

[12] L. Lilburne and S. Tarantola. Sensitivity analysis of spatial models. International Journal of Geographical Information Science, 23:151-168, 2009.

[13] J.C.Helton, J. D. Johnson, J. D. Sallaberry, and C. B. Storlied. Survey of sampling-based methods for uncertainty and sensitivity analysis. Reliability Engineering and System Safety, 91:1175-1209, 2006.

[14] S. Kuchrenko, B. Feil, N. Shah, and W. Mauntz. The identification of model effective dimensions using global sensitivity analysis. Reliability and Engineering Safety, 96:440-449, 2011.

[15] M. Grigoriu. On the spectral representation method in simulation. Probabilistic Engineering Mechanics, 8(2):75-90, 1993.

[16] J. Zhang and B. Ellingwood. Orthogonal series expansions of random processes in reliability analysis. Journal of Engineering Mechanics ASCE, 120(12):2660-2677, 1994. 
[17] S.P. Huang, S. T. Quek, and K. K. Phoon. Convergence study of the truncated Karhunen-Loeve expansion for simulation of stochastic processes. International Journal of Numerical Methods in Engineering, 52(9):1029-1043, 2001.

[18] K. K. Phoon, S. P. Huang, and S. T. Quek. Simulation of second order processes using Karhunen-Loeve expansion. Computers and Structures, 80(12):1049-1060, 2002.

[19] G. Blatman and B. Sudret. Efficient computation of global sensitivity indices using sparse polynomial chaos expansions. Reliability Engineering E5 System Safety, 95(11):1216 - 1229, 2010.

[20] T. Crestaux, O. Le Maitre, and J.M. Martinez. Polynomial chaos expansion for sensitivity analysis. Reliability Engineering \& System Safety, 94:1161-1172, 2009.

[21] B. Sudret. Global sensitivity analysis using polynomial chaos expansion. Reliability Engineering \& System Safety, 93:964-979, 2008.

[22] T. A. Mara and O. Rakoto Joseph. Comparison of some efficient methods to evaluate the main effect of computer model factors. Journal of Statistical Computation and Simulation, 78(2):167-178, 2008.

[23] S. Kucherenko, D. Albrecht, and A. Saltelli. Comparison of Lagin Hypercube and Quasi Monte Carlo sampling techniques. Mathematics and Computers in Simulation, In revision, 2013.

[24] M. D. McKay. Evaluating prediction uncertainty. Technical Report 
NUREG/CR-6311, LA-12915-MS, Statistics Group, Los Alamos National Laboratory, 1995.

[25] R. Ghanem and P. Spanos. Stochastic finite elements - A spectral approach. Springer Verlag, 1991.

[26] K. Maleknejada and T. Lofti, editors. Using Wavelet for Numerical Solution of Fredholm Integral Equations, volume II, London, UK, 2007. World Congress on Engineering, WCE 2007.

[27] J. C. Fort, T. Klein, A. Lagnous, and B. Laurent. Estimation of the Sobol indices in a linear functional multidimensional model. Journal of Statistical Planning and Inference, 143(9):1590-1605, 2013.

[28] F. Gamboa, A. Janon, T. Klein, and A. Lagnoux. Sensitivity indices for multivariate outputs. C. R. Math. Acadmie des Sciences de Paris, 351(7-8):307-310, 2013.

[29] A. Janon, T. Klein, A. Lagnoux, M. Nodet, and C. Prieur. Asymptotic normality and efficiency of two Sobol index estimators. ESAIM: Probability and Statistics, eFirst, 52014.

[30] C. Spitz, L. Mora, E. Wurtz, and A. Jay. Pratical application of uncertainty and sensitivity analysis on an experimental house. Energy and Buildings, 55:459-470, 2012.

[31] J. Goffart, T. Mara, and E. Wurtz. Generation of stochastic weather data for uncertainty and sensitivity analysis of complex energy systems. Applied Energy, In revision, 2013. 
[32] T. A. Mara and S. Tarantola. Application of global sensitivity analysis of model output to building thermal simulations. Journal of Building Simulation, 1:290-302, 2008.

[33] D. Crawley, L. Lawrie, F. Winkelmann, W. Buhl, Y. Huang, C. Pederson, and K. Strand. Energyplus: creating a new generation building energy simulation program. Energy and Buildings, 33(4):319-331, 2001. 


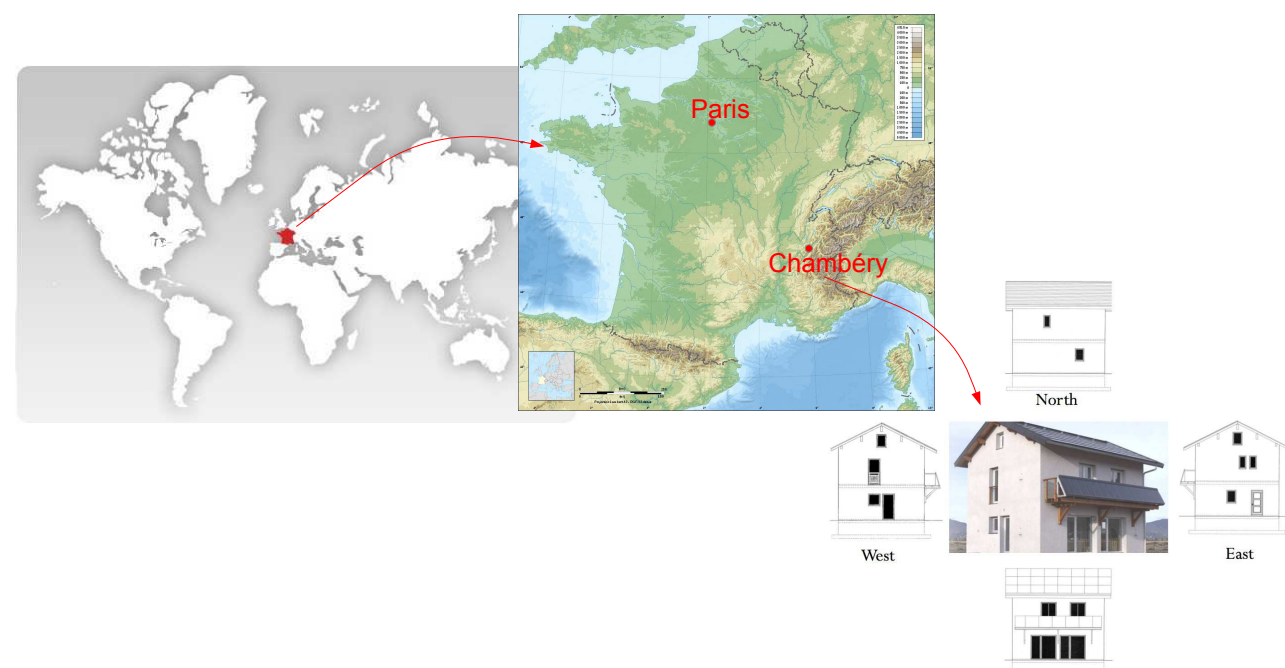

Figure 1: INCAS passive house

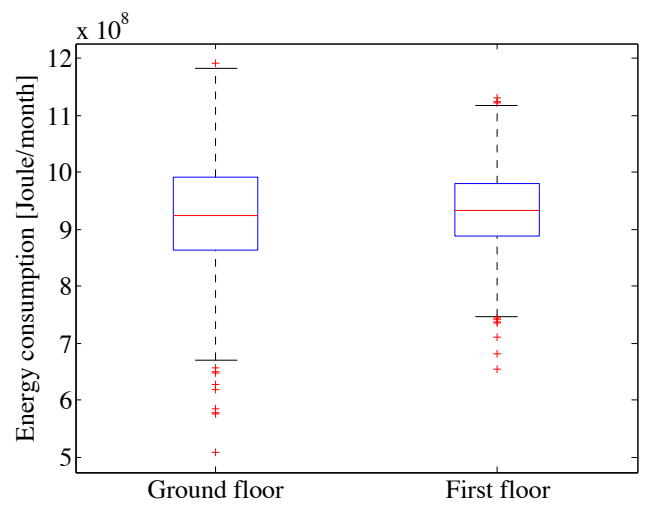

Figure 2: Data dispersion for the heat consumption at the ground and first floors. The central mark in red is the median and the edges of the blue box are the 25th and 75 th percentiles. 


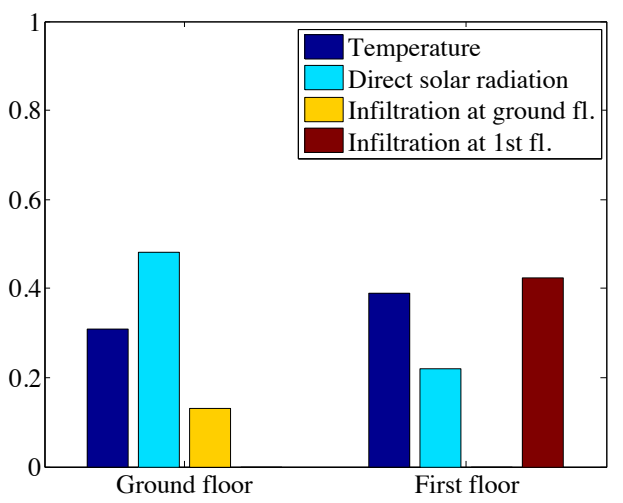

Figure 3: Sensitivity indices for the heat consumption at the ground floor and first floor
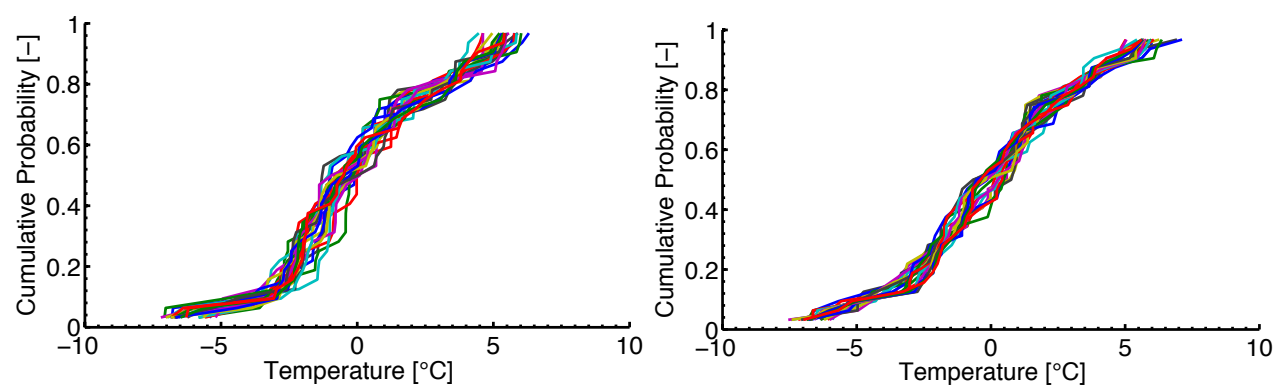

Figure 4: Cumulative distribution function for the outdoor air temperature - left: generated samples, right : source file 

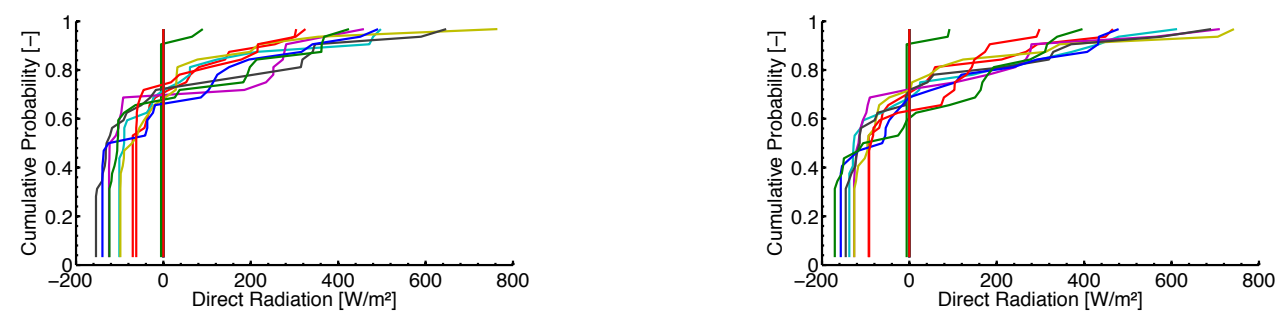

Figure 5: Cumulative distribution function for the direct solar radiation - left: generated samples, right : source file
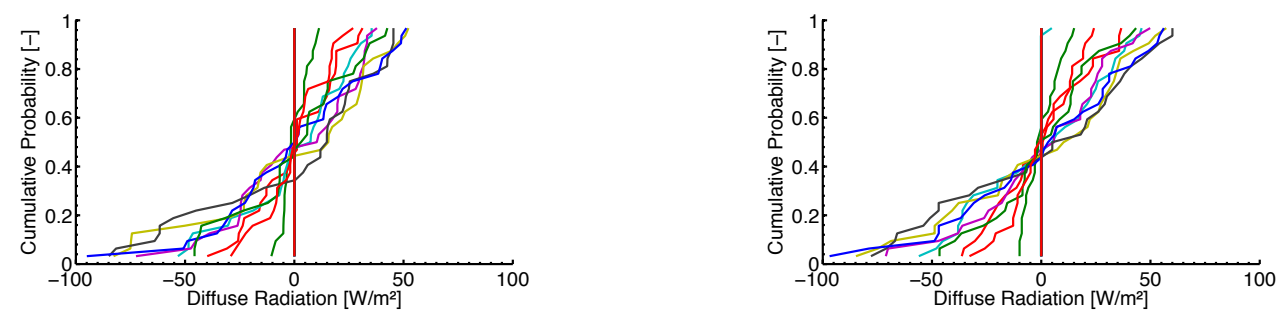

Figure 6: Cumulative distribution function for the diffuse solar radiation - left: generated samples, right : source file
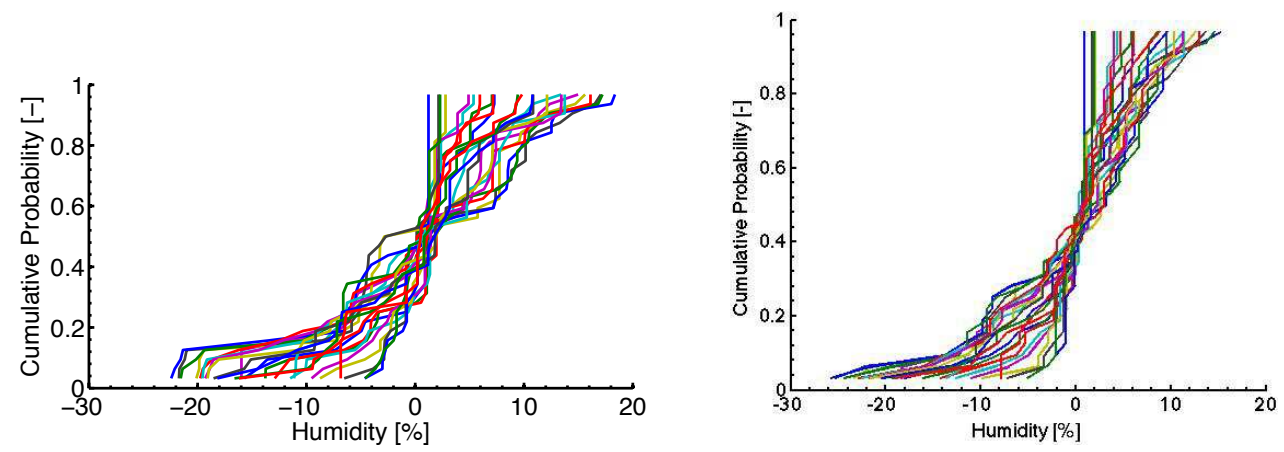

Figure 7: Cumulative distribution function for the relative humidity - left: generated samples, right : source file 

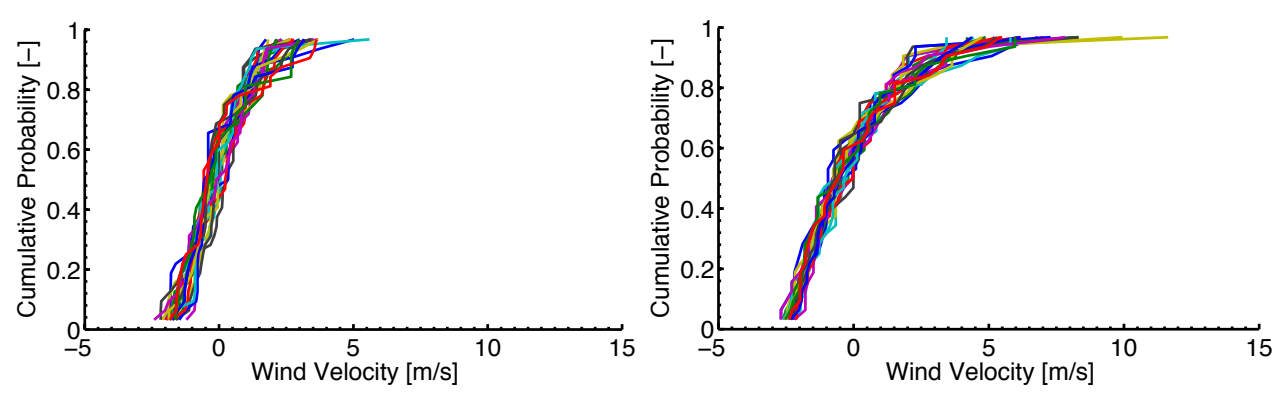

Figure 8: Cumulative distribution function for the wind speed - left: generated samples, right : source file
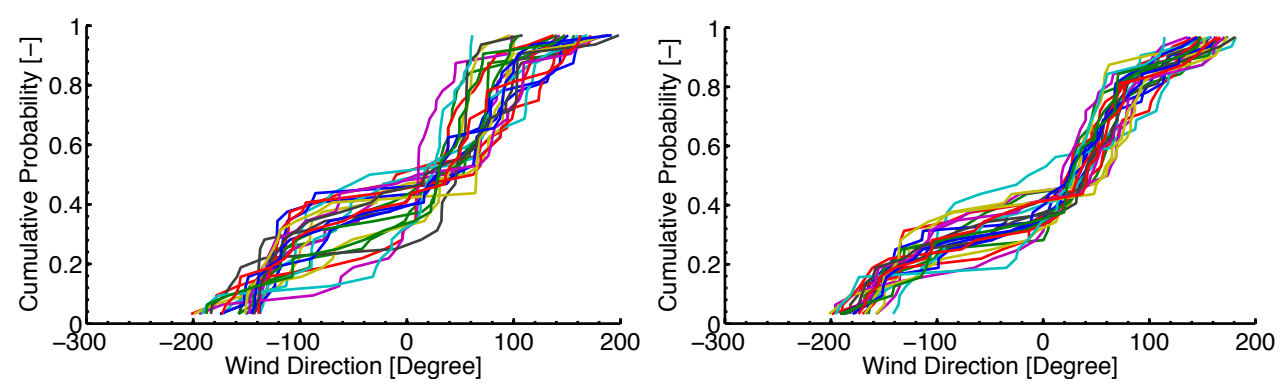

Figure 9: Cumulative distribution function for the wind direction - left: generated samples, right : source file

\section{Appendix A. List of uncertain static inputs}




\begin{tabular}{|c|c|c|}
\hline Building Components & Physical Quantity & Distribution Characteristics \\
\hline Glass Wool (35) & Conductivity $\left[\mathrm{W} \cdot \mathrm{m}^{-1} \cdot \mathrm{K}^{-1}\right]$ & $\mathcal{N}(0.035,0.00175)$ \\
\hline Outdoor wall insulation & Density $\left[\mathrm{kg} \cdot \mathrm{m}^{-3}\right]$ & $\mathcal{N}(12,0.6)$ \\
\hline Glass Wool (32) & Conductivity $\left[\mathrm{W} \cdot \mathrm{m}^{-1} \cdot \mathrm{K}^{-1}\right]$ & $\mathcal{N}(0.032,0.0016)$ \\
\hline $\begin{array}{l}\text { Outdoor wall insulation of } \\
\text { the attic }\end{array}$ & Density $\left[\mathrm{kg} \cdot \mathrm{m}^{-3}\right]$ & $\mathcal{N}(12,0.6)$ \\
\hline Glass Wool (35) & Conductivity $\left[\mathrm{W} \cdot \mathrm{m}^{-1} \cdot \mathrm{K}^{-1}\right]$ & $\mathcal{N}(0.035,0.00175)$ \\
\hline $\begin{array}{l}\text { Insulation between First } \\
\text { floor - Attic }\end{array}$ & Density $\left[\mathrm{kg} \cdot \mathrm{m}^{-3}\right]$ & $\mathcal{N}(12,0.6)$ \\
\hline Extruded Polystyrene Foam & Conductivity $\left[\mathrm{W} \cdot \mathrm{m}^{-1} \cdot \mathrm{K}^{-1}\right]$ & $\mathcal{N}(0.03,0.0015)$ \\
\hline $\begin{array}{l}\text { Insulation Crawlspace- } \\
\text { Ground floor }\end{array}$ & Density $\left[\mathrm{kg} \cdot \mathrm{m}^{-3}\right]$ & $\mathcal{N}(35,1.75)$ \\
\hline Extruded Polystyrene Foam & Conductivity $\left[\mathrm{W} \cdot \mathrm{m}^{-1} \cdot \mathrm{K}^{-1}\right]$ & $\mathcal{N}(0.029,0.00145)$ \\
\hline Insulation of the Attic walls & Density $\left[\mathrm{kg} \cdot \mathrm{m}^{-3}\right]$ & $\mathcal{N}(35,1.75)$ \\
\hline Concrete blocks & Conductivity $\left[\mathrm{W} \cdot \mathrm{m}^{-1} \cdot \mathrm{K}^{-1}\right]$ & $\mathcal{N}(1,0.05)$ \\
\hline Wall & Density $\left[\mathrm{kg} . \mathrm{m}^{-3}\right]$ & $\mathcal{N}(1100,55)$ \\
\hline Concrete Floor & Conductivity $\left[\mathrm{W} \cdot \mathrm{m}^{-1} \cdot \mathrm{K}^{-1}\right]$ & $\mathcal{N}(2.75,0.1375)$ \\
\hline Ground Floor & Density $\left[\mathrm{kg} \cdot \mathrm{m}^{-3}\right]$ & $\mathcal{N}(2400,120)$ \\
\hline Slab $(16 \mathrm{~cm})$ & Conductivity $\left[\mathrm{W} \cdot \mathrm{m}^{-1} \cdot \mathrm{K}^{-1}\right]$ & $\mathcal{N}(2.5,0.125)$ \\
\hline Floor of the Ground Floor & Density $\left[\mathrm{kg} \cdot \mathrm{m}^{-3}\right]$ & $\mathcal{N}(2400,120)$ \\
\hline Hollow-core slab & Conductivity $\left[\mathrm{W} \cdot \mathrm{m}^{-1} \cdot \mathrm{K}^{-1}\right]$ & $\mathcal{N}(1.23,0.0615)$ \\
\hline Floor of the First Floor & Density $\left[\mathrm{kg} \cdot \mathrm{m}^{-3}\right]$ & $\mathcal{N}(1300,65)$ \\
\hline Concrete Floor & Conductivity $\left[\mathrm{W} \cdot \mathrm{m}^{-1} \cdot \mathrm{K}^{-1}\right]$ & $\mathcal{N}(2.75,0.1375)$ \\
\hline Floor of the First Floor & Density $\left[\mathrm{kg} \cdot \mathrm{m}^{-3}\right]$ & $\mathcal{N}(2400,120)$ \\
\hline Slab $(4 \mathrm{~cm})$ & Conductivity $\left[\mathrm{W} \cdot \mathrm{m}^{-1} \cdot \mathrm{K}^{-1}\right]$ & $\mathcal{N}(2.5,0.125)$ \\
\hline Floor of the First Floor & Density $\left[\mathrm{kg} \cdot \mathrm{m}^{-3}\right]$ & $\mathcal{N}(2400,120)$ \\
\hline
\end{tabular}




\begin{tabular}{|c|c|c|}
\hline Building Components & Physical Quantity & Distribution Characteristics \\
\hline Floor Tile & Conductivity $\left[\mathrm{W} \cdot \mathrm{m}^{-1} \cdot \mathrm{K}^{-1}\right]$ & $\mathcal{N}(0.41,0.0205)$ \\
\hline Floor of the Ground Floor & Density $\left[\mathrm{kg} . \mathrm{m}^{-3}\right]$ & $\mathcal{N}(1200,60)$ \\
\hline Coating & Conductivity $\left[\mathrm{W} \cdot \mathrm{m}^{-1} \cdot \mathrm{K}^{-1}\right]$ & $\mathcal{N}(1,0.05)$ \\
\hline Outdoor walls & Density $\left[\mathrm{kg} \cdot \mathrm{m}^{-3}\right]$ & $\mathcal{N}(1450,72.5)$ \\
\hline Plaster & Conductivity $\left[\mathrm{W} \cdot \mathrm{m}^{-1} \cdot \mathrm{K}^{-1}\right]$ & $\mathcal{N}(0.43,0.0215)$ \\
\hline Indoor walls & Density $\left[\mathrm{kg} \cdot \mathrm{m}^{-3}\right]$ & $\mathcal{N}(1200,60)$ \\
\hline Dropped Ceiling & Conductivity $\left[\mathrm{W} \cdot \mathrm{m}^{-1} \cdot \mathrm{K}^{-1}\right]$ & $\mathcal{N}(0.35,0.0175)$ \\
\hline The First Floor and Ground & Density $\left[\mathrm{kg} \cdot \mathrm{m}^{-3}\right]$ & $\mathcal{N}(36.9,1.845)$ \\
\hline Floor ceilings & & \\
\hline Roof Tile & Conductivity $\left[\mathrm{W} \cdot \mathrm{m}^{-1} \cdot \mathrm{K}^{-1}\right]$ & $\mathcal{N}(2.2,0.11)$ \\
\hline Roof & Density $\left[\mathrm{kg} \cdot \mathrm{m}^{-3}\right]$ & $\mathcal{N}(2700,135)$ \\
\hline Air & Conductivity $\left[\mathrm{W} \cdot \mathrm{m}^{-1} \cdot \mathrm{K}^{-1}\right]$ & $\mathcal{N}(0.025,0.00125)$ \\
\hline (in the dropped ceiling) & Density $\left[\mathrm{kg} \cdot \mathrm{m}^{-3}\right]$ & $\mathcal{N}(1.23,0.0615)$ \\
\hline Gravel & Conductivity $\left[\mathrm{W} \cdot \mathrm{m}^{-1} \cdot \mathrm{K}^{-1}\right]$ & $\mathcal{N}(2,0.1)$ \\
\hline Surrounding Soil & Density $\left[\mathrm{kg} \cdot \mathrm{m}^{-3}\right]$ & $\mathcal{N}(1600,80)$ \\
\hline Ground Temperature & Ground Temperature $\left[{ }^{\circ} \mathrm{C}\right]$ & $\mathcal{N}(7,1,5)$ \\
\hline \multirow[t]{2}{*}{ South Window } & Conductivity $\left[\mathrm{W} \cdot \mathrm{m}^{-1} \cdot \mathrm{K}^{-1}\right]$ & $\mathcal{N}(1.1,0.055)$ \\
\hline & Transmittance [-] & $\mathcal{N}(0.6,0.03)$ \\
\hline \multirow[t]{2}{*}{ East Window } & Conductivity $\left[\mathrm{W} \cdot \mathrm{m}^{-1} \cdot \mathrm{K}^{-1}\right]$ & $\mathcal{N}(1.1,0.055)$ \\
\hline & Transmittance [-] & $\mathcal{N}(0.6,0.03)$ \\
\hline \multirow[t]{2}{*}{ West Window } & Conductivity $\left[\mathrm{W} \cdot \mathrm{m}^{-1} \cdot \mathrm{K}^{-1}\right]$ & $\mathcal{N}(1.1,0.055)$ \\
\hline & Transmittance [-] & $\mathcal{N}(0.6,0.03)$ \\
\hline \multirow{2}{*}{$\begin{array}{l}\text { North Window } \\
\text { (triple glazed window) }\end{array}$} & Conductivity $\left[\mathrm{W} \cdot \mathrm{m}^{-1} \cdot \mathrm{K}^{-1}\right]$ & $\mathcal{N}(0.7,0.035)$ \\
\hline & Transmittance [-] & $\mathcal{N}(0.45,0.0225)$ \\
\hline
\end{tabular}




\begin{tabular}{|l|l|l|}
\hline Building Components & Physical Quantity & Distribution Characteristics \\
\hline \hline Albedo & Albedo [-] & $\mathcal{U}(0.27,0.33)$ \\
\hline Orientation & $\begin{array}{l}\text { Degrees from true North } \\
{[\text { Degree] }}\end{array}$ & $\mathcal{N}(-15,1.5)$ \\
\hline Infiltration Rate & Infiltration Rate $[\mathrm{Vol} / \mathrm{h}]$ & $\mathcal{N}(2,0.5)$ \\
Crawlspace & Infiltration Rate $[\mathrm{Vol} / \mathrm{h}]$ & $\mathcal{N}(2,0.5)$ \\
Attic & Infiltration Rate $[\mathrm{Vol} / \mathrm{h}]$ & $\mathcal{N}(0.115,0.29)$ \\
First Floor & Infiltration Rate $[\mathrm{Vol} / \mathrm{h}]$ & $\mathcal{N}(0.115,0.29)$ \\
Ground Floor & \multicolumn{2}{|l}{} \\
\hline
\end{tabular}

Table A.1: List of uncertain inputs 